\title{
Perioperative Hemodynamic Monitoring
}

\section{An Overview of Current Methods}

\author{
Ilonka N. de Keijzer, MD*, Thomas W.L. Scheeren, MD, PhD
}

\section{KEYWORDS}

- Hemodynamic monitoring • Blood pressure - Mean systemic filling pressure

- Central venous pressure $\bullet$ Cardiac output monitoring • Fluid responsiveness

- Volume monitoring

\section{KEY POINTS}

- The aim of hemodynamic management is to optimize the amount of oxygen delivered to tissues.

- Because direct monitoring of the amount of oxygen delivered remains difficult, hemodynamic variables are monitored instead.

- Hemodynamic monitoring itself does not improve patient outcomes and needs to be combined with treatment protocols.

- Pressures, from arterial blood pressure through pulmonary artery occlusion pressure, can be measured using invasive catheters, but are subject to artifacts (e.g. over- and underdamping, patient movements) and should be zeroed correctly before adequate measurements can be obtained.

- Flow, that is cardiac output, is mostly obtained by indicator dilution techniques or pulse wave analysis. The evidence for using other noninvasive techniques, for example pulse wave transit time, bioimpedance and bioreactance, is limited.

\section{INTRODUCTION}

The word "hemodynamic" is derived from the Greek words haima and dunamikós. Hemodynamic monitoring, therefore, freely translates into observing the motion of blood. As the word itself, hemodynamic monitoring originates from ancient times: Feeling the pulse was first described in 2600 BCE in the myth of a Mesopotamian king whose friend had died, and by "touching" his heart, he realized that it did not beat any more, ${ }^{1}$ demonstrating that at that time mankind understood the heart was beating and that its pulsations could be felt. Two thousand years later, Hippocrates described

\footnotetext{
Department of Anesthesiology, University Medical Center Groningen, Hanzeplein 1, PO Box 30. 001, 9700 RB Groningen, The Netherlands

* Corresponding author.

E-mail address: i.n.de.keijzer@umcg.nl
} 
pulse characteristics during different states of disease, while Praxagoras (born around 340 BCE) was the first one to use the pulse for indication of disease. ${ }^{2}$ The actual circulation was first described by William Harvey in the seventeenth century, and this was considered one of the greatest contributions to the field of cardiovascular science. ${ }^{3}$ Further development of measuring hemodynamic variables took until 1733, when Stephen Hales pioneered measuring intra-arterial pressure in horses ${ }^{4}$ and this discovery was followed by the development of the "Stromuhr" in 1867 by Carl Ludwig, a device able to quantify blood flow through perfused organs. ${ }^{5}$ From there on forward, important discoveries followed more rapidly, including the first noninvasive systolic blood pressure measuring device using a cuff-based version of the mercury sphygmograph by Riva Rocci in $1896 .{ }^{6}$ In 1905 , Nicolai Korotkoff described the sounds auscultating the brachial artery while deflating the cuff. ${ }^{7}$ This way, it became possible to also determine diastolic blood pressure. Around the same time, in 1901, Willem Einthoven invented the electrocardiograph for which he later (1924) received the Nobel Prize..$^{8,9}$ The foundation for modern hemodynamic monitoring was laid.

\section{PRESSURE MONITORING Arterial Blood Pressure}

Arterial blood pressure was one of the first hemodynamic variables that could be measured. ${ }^{7}$ Currently, different techniques are available for continuous and intermittent arterial blood pressure monitoring in the perioperative period. Arterial catheterization is the gold standard for measuring blood pressure continuously. ${ }^{10} \mathrm{~A}$ catheter is placed into a superficial artery (mostly radial artery) and is connected via a fluid filled tube to a pressure transducer and a pressurized bag of fluids which creates counterpressure for the arterial pressure. ${ }^{11}$ The pressure transducer transforms mechanical pressure into an electrical signal, which is used to depict the arterial pressure waveform on a monitor. The arterial pressure waveform is the result of the interaction between the left ventricle and the systemic arteries. The pressure sensor should be placed at right atrium level and zeroed against atmospheric pressure to obtain reliable measurements. Over- and underdamping can underestimate or overestimate arterial blood pressure. ${ }^{11}$ Additionally, blood pressure measurements can be influenced by movement of the patient's arm or a kink in the pressure system. ${ }^{10,11}$ The use of invasive blood pressure monitoring is limited owing to the risk of complications and costs associated with arterial catheterization. ${ }^{12,13}$ Frequently used alternative techniques for invasive blood pressure monitoring are noninvasive intermittent oscillometry and the continuous volume clamp method. ${ }^{14}$ The most widely used method of measuring arterial blood pressure noninvasively is oscillometry. Usually, a brachial cuff is inflated and instead of Korotkoff sounds, oscillations of the blood pressure signal are detected. ${ }^{15}$ The pressure which causes maximum oscillations is closest to the mean arterial pressure (MAP), and systolic and diastolic blood pressures are mathematically derived from this mean value. ${ }^{16}$ The noninvasive volume clamp method uses a plethysmograph and an inflatable finger cuff. The plethysmograph detects the blood volume in the digital arteries and subsequently the pressure from the inflatable finger cuff is adjusted with high frequency to keep the blood volume in the digital arteries constant (volume clamp). Subsequently, the arterial pressure waveform can be constructed from the amount of pressure in the finger cuff needed to ensure constant volume. ${ }^{17-19}$ Calibration is periodically performed by applying a constant pressure, to determine MAP and subsequently calibrate the plethysmography signal. ${ }^{14}$ The use of the volume clamp method has also been validated in obese patients ${ }^{20}$ and in patients with atrial fibrillation ${ }^{21}$; however, the use is limited in patients with impaired peripheral perfusion, 
for example in patients receiving high doses of vasopressors or during peripheral hypothermia or edema. ${ }^{14,21}$ Other methods for measuring blood pressure noninvasively include applanation tonometry, hydraulic coupling, pulse wave transit time, and pulse decomposition. The evidence for the accuracy of these methods is limited and therefore these are not (yet) widely used in daily clinical routine. ${ }^{10}$

\section{Central Venous Pressure}

The central venous pressure (CVP) is the pressure measured in the vena cava near the right atrium, and is commonly obtained by placing a central venous catheter in the superior vena cava via the internal jugular vein or the subclavian vein. ${ }^{22}$ The catheter is then connected to a pressure transducer via a fluid filled line. The CVP is determined by cardiac function and venous return to the heart, ${ }^{23,24}$ and has traditionally been used to estimate preload and volume status, ${ }^{23,25}$ and also to guide fluid therapy. ${ }^{26}$ It was assumed that, because obtaining ventricular end-diastolic volume was not suitable in many clinical settings, the CVP would be a surrogate measure of preload. ${ }^{25} \mathrm{Howev}-$ er the use of the CVP as a surrogate for end-diastolic volume was questioned owing to the poor correlation with the volume status of the patient ${ }^{25,27-29}$ and should no longer be used to assess fluid responsiveness. ${ }^{29,30}$ Nonetheless, the CVP is still measured frequently and might be used instead as an indication to stop fluid resuscitation. ${ }^{31}$ Although the CVP is not able to predict fluid responsiveness, it can be used to assess right ventricular function, for example, in pulmonary embolism, right ventricular failure, or after heart transplantation. ${ }^{29}$ Another advantage from using a central venous catheter is the possibility of taking blood gas samples for measuring the central venous oxygen saturation. ${ }^{32}$

\section{Pulmonary Artery Pressures}

Pulmonary artery pressures include the systolic and diastolic pressures and the pulmonary artery occlusion pressure (PAOP) and can be assessed by a pulmonary artery catheter (PAC). ${ }^{33,34}$ A PAC is inserted via the internal jugular vein, subclavian vein, or femoral vein through the right atrium and right ventricle until its tip is positioned in the pulmonary artery. ${ }^{34}$ The catheter is then connected via a tubing to a pressure transducer to obtain pressure measurements. These pressure measurements can be used during insertion to assess the position of the tip. The catheter contains 2 or more ports; the distal port is located at the tip and the other port is located more proximal and can be used to measure the CVP. ${ }^{34}$ A PAC contains a balloon close to the tip, which can be inflated to float the catheter into the pulmonary artery and to determine PAOP. ${ }^{33}$ The PAOP reflects the pressure in the pulmonary veins and left atrium. ${ }^{33,35}$ In analogy to the CVP for the right ventricle, the PAOP was used as a predictor of left ventricular preload, but turned out to be unreliable for this purpose as well. ${ }^{25}$ The use of a PAC can be associated with several (severe) complications, ${ }^{33}$ and treatment benefits of using a PAC have not been clearly established in critically ill patients. ${ }^{36,37}$ Therefore, the evidence for using a PAC has been questioned. The use of a PAC should always be combined with a specific treatment protocol to improve patient outcomes. ${ }^{38}$ The PAC is still frequently used worldwide, particularly in cardiac surgery, in patients with pulmonary arterial hypertension (suspected or known), severe cardiogenic shock, unknown volume status in shock, or other severe cardiopulmonary disease. ${ }^{39-41}$ It has been suggested that clinicians use the PAC to gain a clear understanding of the pathophysiology. ${ }^{42}$ Similar to the central venous catheter, a PAC can be used to obtain blood samples for measuring mixed venous oxygen saturation, a marker of the global relation between oxygen delivery and consumption or the ability of the tissues to extract oxygen from the blood. 


\section{Mean Systemic Filling Pressure}

The mean systemic filling pressure $\left(P_{\mathrm{msf}}\right)$ is the pressure that equilibrates in the systemic circulation when the heart stops pumping and all blood is distributed equally throughout the systemic circulation. ${ }^{43}$ The value of the $P_{\mathrm{msf}}$ is therefore between MAP and CVP (closer to the latter owing to the size of the venous blood reservoir). The $P_{\mathrm{msf}}$ does not include the pressures in the pulmonary circulation and cardiac chambers (mean cardiopulmonary filling pressure). The $P_{\mathrm{msf}}$ and mean cardiopulmonary filling pressure combined are the mean circulatory filling pressure. ${ }^{43}$ The $P_{\text {msf }}$ resembles the stressed blood volume, that is, the amount of blood that exerts pressure against the vascular walls. The unstressed blood volume in turn is the amount of blood, which can be held within the vascular system without creating pressure. ${ }^{43}$ The $P_{\text {msf }}$ can be measured in different ways: (1) by the inspiratory hold method, (2) by the arm stop-flow method, or (3) through a calculated model. ${ }^{44,45}$ The inspiratory hold method uses inspiratory holds of several seconds at plateau pressure to briefly increase the CVP. When the CVP increases, the venous return decreases and consequently the cardiac output (CO) decreases as well. Multiple inspiratory hold maneuvers at different plateau pressure levels are performed to derive pairs of $\mathrm{CO}$ and CVP measurements, which are then correlated and extrapolated to zero CO (which resembles a no flow state) and the pressure that remains is the estimated $P_{\text {msf }}$ (Fig. 1). For this method, a central venous catheter and $\mathrm{CO}$ monitoring are required. ${ }^{46} \mathrm{Howev}-$ er, the $P_{\text {msf }}$ might be overestimated by this method because high airway pressures may redistribute blood from the pulmonary to the systemic circulation. ${ }^{47}$ The arm stop-flow method is performed using a rapidly inflating arm cuff, which occludes

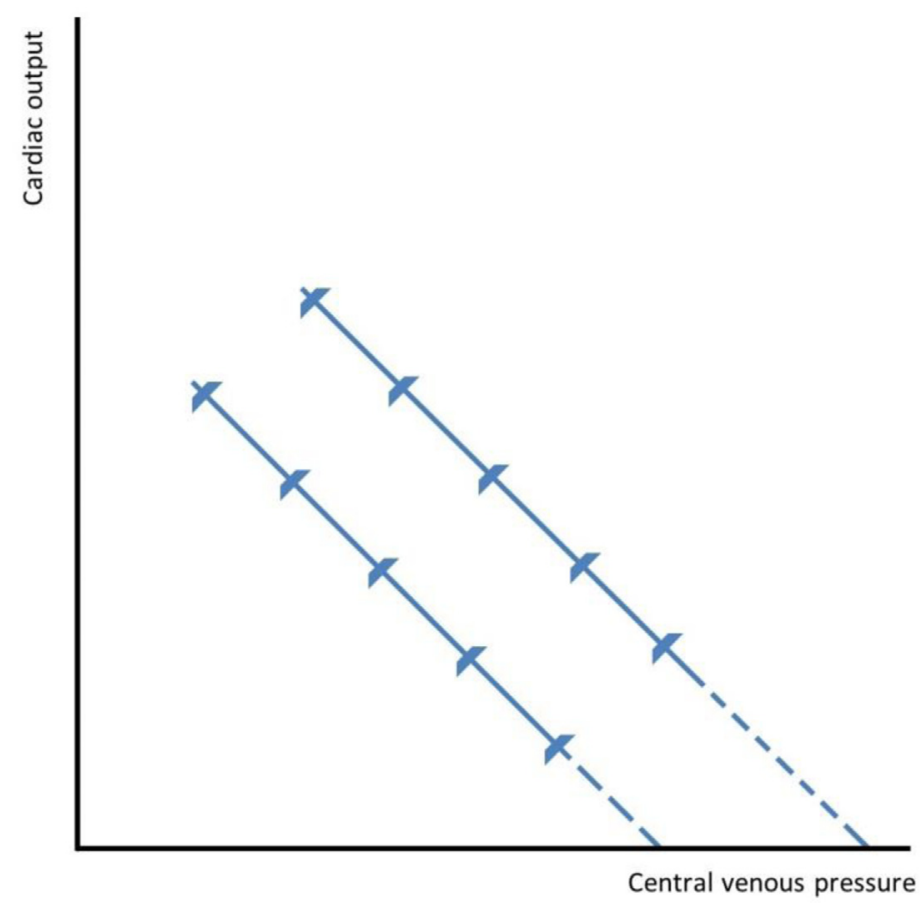

Fig. 1. Venous return curves plotted using the inspiratory hold method for different volume states. The right/upper curve is the higher volume state. Inspiratory hold maneuver. 
the arteries in the arm creating a status of zero flow. The intravascular pressure will equilibrate between the venous and arterial compartment after approximately 30 seconds. The equilibration pressure is an estimation of the $P_{\text {msf }}$. For this method, only an arterial catheter is required. ${ }^{44}$ The last method uses a mathematical model to estimate the $P_{\text {msf }}$. The $P_{\text {msf }}$ comprises of the arterial and venous compartment and resistance to flow, resulting in the following formula: $P_{\text {msf }}=a \mathrm{RAP}+b \mathrm{MAP}+c \mathrm{CO}$, in which RAP is right atrial pressure, MAP is mean arterial pressure, and $\mathrm{CO}$ is cardiac output ${ }^{48}, a$ and $b$ are both dimensionless constants (often $a=0.96, b=0.04$ ), reflecting the contribution of venous and arterial blood, and $c$ is a constant determined by age, height, and weight, resembling resistance. ${ }^{48}$ The $P_{\text {msf }}$ can be used to accurately assess volume status, although it is quite difficult to measure and thus of limited clinical use. Additionally, the $P_{\text {msf }}$ minus the right atrial pressure is the driving force for venous return. ${ }^{49} \mathrm{An}$ overview of all pressure monitoring methods can be found in Table 1.

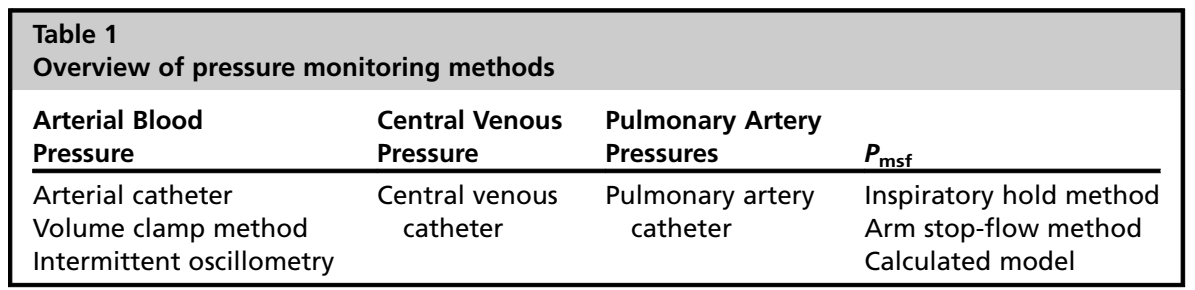

\section{FLOW MONITORING \\ Cardiac Output}

\section{Invasive cardiac output monitoring}

The $\mathrm{CO}$ is the product of stroke volume and heart rate and is the primary determinant of oxygen delivery to organs and peripheral tissues, therefore being one of the most clinically relevant hemodynamic variables. Invasive methods of determining $\mathrm{CO}$ include pulmonary artery thermodilution (PATD), transpulmonary thermodilution (TPTD), and lithium dilution. ${ }^{50}$ All of these techniques use modified versions of the Stewart Hamilton equation to determine CO. ${ }^{51}$ The Stewart Hamilton equation is based on the fact that, if the volume and temperature or concentration of an injected indicator are known, then the change in temperature or indicator concentration downstream is related to the flow, that is $\mathrm{CO}$, and can be calculated as follows:

$$
C O=\frac{\text { Temperature of indicator }}{\text { Area under the temperature curve }}
$$

PATD requires a PAC, through which a cold fluid bolus can be injected to the right atrium and downstream temperature changes can be measured at the tip thermistor. Because the tip of the PAC is located in the pulmonary artery, this method actually measures right ventricular output. ${ }^{50,51}$ Newer generation PACs incorporate electric heating elements to continuously assess temperature differences downstream and, thus, CO. ${ }^{52}$ TPTD requires a central venous catheter and an arterial catheter placed in a systemic artery in proximity to the heart. ${ }^{51}$ Using this technique, a cold fluid bolus is injected in the central venous circulation and the blood temperature difference is measured in the systemic circulation, therefore measuring the global CO. Unlike PATD, TPTD is not influenced by the ventilatory cycle. ${ }^{52}$ The use of both methods is limited in patients with intracardiac shunts, low-flow states, or tricuspid 
regurgitation. ${ }^{52}$ For lithium dilution, a bolus of lithium chloride solution is injected in a peripheral or central vein, and the systemic lithium concentration is detected downstream by an electrode sensor implanted in the arterial catheter. The lithium dilution method for assessing $\mathrm{CO}$ is limited in patients treated with lithium and is contraindicated in the first trimester of pregnancy. Additionally, lithium boluses cannot be given frequently, because of lithium accumulation. ${ }^{52}$ All these indicator dilution techniques are primarily used in patients undergoing cardiac surgery. ${ }^{50}$

\section{Minimally invasive cardiac output monitoring}

Minimally invasive $\mathrm{CO}$ measurement methods use a peripheral arterial catheter to obtain the arterial pressure waveform. By analyzing this waveform an estimation of the stroke volume and, thus, the $\mathrm{CO}$ can be made. ${ }^{50}$ There are calibrated and uncalibrated minimally invasive methods to estimate CO. Calibrated methods need an externally derived CO value, that is one obtained by PATD or TPTD, to adjust the estimated $\mathrm{CO}$. Uncalibrated methods estimate $\mathrm{CO}$ solely based on characteristics of the arterial pressure waveform ${ }^{53,54}$ in combination with demographic factors. Pulse wave analysis is indicated in high-risk patients or in patients who are planned to undergo a high-risk procedure. ${ }^{50}$ Calibrated methods are preferred in patients with low systemic vascular resistance, such as liver disease and sepsis. ${ }^{55}$ The use of pulse wave analysis is limited in patients with frequent and fast changes in vascular tone and in patients with cardiac arrhythmias. ${ }^{50}$ Naturally, the quality of the arterial pressure waveform signal is of crucial importance to the reliability of the derived $\mathrm{CO}$ estimates. ${ }^{50}$

\section{Noninvasive cardiac output monitoring}

Multiple methods are available for assessing $\mathrm{CO}$ noninvasively including volume clamp method, electrical bioimpedance, thoracic bioreactance, and pulse wave transit time. ${ }^{56}$ As explained elsewhere in this article, the volume clamp method obtains the arterial pressure waveform noninvasively from which the stroke volume and thus $\mathrm{CO}$ can be derived. Electrical bioimpedance is based on the assumption that resistance to electrical current (impedance) changes during the cardiac cycle owing to the fluctuating blood volume in the thorax. These changes can be quantified by measuring the changes in voltages from the applied and detected current via surface electrodes to estimate $\mathrm{CO}$. In the majority of studies it has been shown that the $\mathrm{CO}$ estimates by electrical bioimpedance lack accuracy and precision. ${ }^{56-59}$ Additionally, the use of bioimpedance is limited in patients with cardiac arrhythmias, ${ }^{60}$ pathologic accumulation of fluid in the thorax, ${ }^{61}$ and during electrical interference (such as electrocautery). A new device was developed that used electrodes attached to an endotracheal tube to obtain the bioimpedance signal closer to the source, that is, the ascending aorta. ${ }^{62}$ Thoracic bioreactance is a newer technology also based on the principle of bioimpedance. However, bioreactance focuses on the phase shift of the bioimpedance signal. The phase shift occurs owing to pulsatile flow, primarily coming from the aorta. Because only pulsatile flow is accounted for, pathologic fluid collections in the thorax do not affect these measurements. ${ }^{63}$ However, thoracic bioreactance is also disturbed by electrical interference, and the agreement between bioreactance and reference methods was poor. ${ }^{56}$ The pulse wave transit time is defined as the time that the stroke volume travels from the heart to the periphery, and can be measured as the time difference between the $R$ top in the electrocardiogram and the start of the plethysmographic waveform obtained by pulse oximetry. ${ }^{56}$ The pulse wave transit time is inversely correlated with stroke volume and thus the $\mathrm{CO}$; that is it decreases when stroke volume and $\mathrm{CO}$ increase. Evidence for the use of the pulse 
wave transit time method is limited. Currently, the volume clamp method is the most studied one of the methods described in this paragraph. ${ }^{18}$ An overview of all CO monitoring methods can be found in Table 2.

\section{VOLUME MONITORING}

Besides measuring blood pressure and blood flow, and in contrast with measuring total blood volume, which is highly complex, several compartmental volumes with clinical relevance can be measured with sufficient accuracy. These include GEDV and extravascular lung water (EVLW).

\section{Global End-Diastolic Volume}

The GEDV is the amount of blood volume present in all heart chambers at the end of the diastole. During diastole the heart is passively filled with blood and thus the GEDV resembles cardiac preload. ${ }^{27}$ TPTD can be used to calculate the GEDV ${ }^{51}$ as the difference between the intrathoracic thermal volume (ie, total intrathoracic volume) and the pulmonary thermal volume (ie, pulmonary volume and volume of the pulmonary circulation) (Fig. 2). Intrathoracic thermal volume is calculated by multiplying $\mathrm{CO}$ with the mean transit time, that is the time from injection of the indicator until one-half of the indicator passes the detection point. The pulmonary thermal volume is calculated as the CO multiplied by the downslope time of the natural log-transformed blood temperature curve measured in a systemic artery. ${ }^{51}$ Because the GEDV is a static variable, it is known to be a less reliable predictor of fluid responsiveness compared with dynamic indices (as discussed elsewhere in this article). ${ }^{64}$ However, the GEDV can be used to assess whether the cardiac preload adequately increases during volume loading.

\section{Extravascular Lung Water}

Fluid accumulated in the extravascular space within the lungs, that is in the interstitial space and alveoli, is called the EVLW. Fluid can leak from the capillaries owing to increased hydrostatic pressure (fluid overload) or increased lung permeability (acute respiratory distress syndrome). ${ }^{65}$ TPTD can be used to estimate the EVLW by subtracting the intrathoracic blood volume from the intrathoracic thermal volume. ${ }^{51}$ The intrathoracic blood volume can be estimated by multiplying the GEDV by $1.25 .^{66}$ EVLW can aid in the diagnosis and severity assessment of pulmonary edema and acute respiratory distress syndrome and has been shown to predict adverse outcome. ${ }^{66}$ The estimation of EVLW by TPTD is compromised by major pulmonary embolism, in patients with a partial lung resection, and in patients with significant pleural effusion. ${ }^{51}$ Fig. 2 gives a graphical display of all volumetric calculations.

\section{FLUID RESPONSIVENESS}

Fluid responsiveness is mostly defined as an increase in stroke volume or CO of $10 \%$ to $15 \%$ after the administration of a fluid bolus. ${ }^{67}$ Because poor outcomes are

\begin{tabular}{l}
$\begin{array}{l}\text { Table } 2 \\
\text { Overview of CO monitoring methods }\end{array}$ \\
\begin{tabular}{lll} 
Invasive Methods & Minimally Invasive Methods & Noninvasive Methods \\
\hline PATD & Pulse wave analysis & Volume clamp method \\
TPTD & (calibrated and uncalibrated) & Electrical bioimpedance \\
Lithium dilution & & Thoracic bioreactance \\
& & Pulse wave transit time
\end{tabular} \\
\hline
\end{tabular}




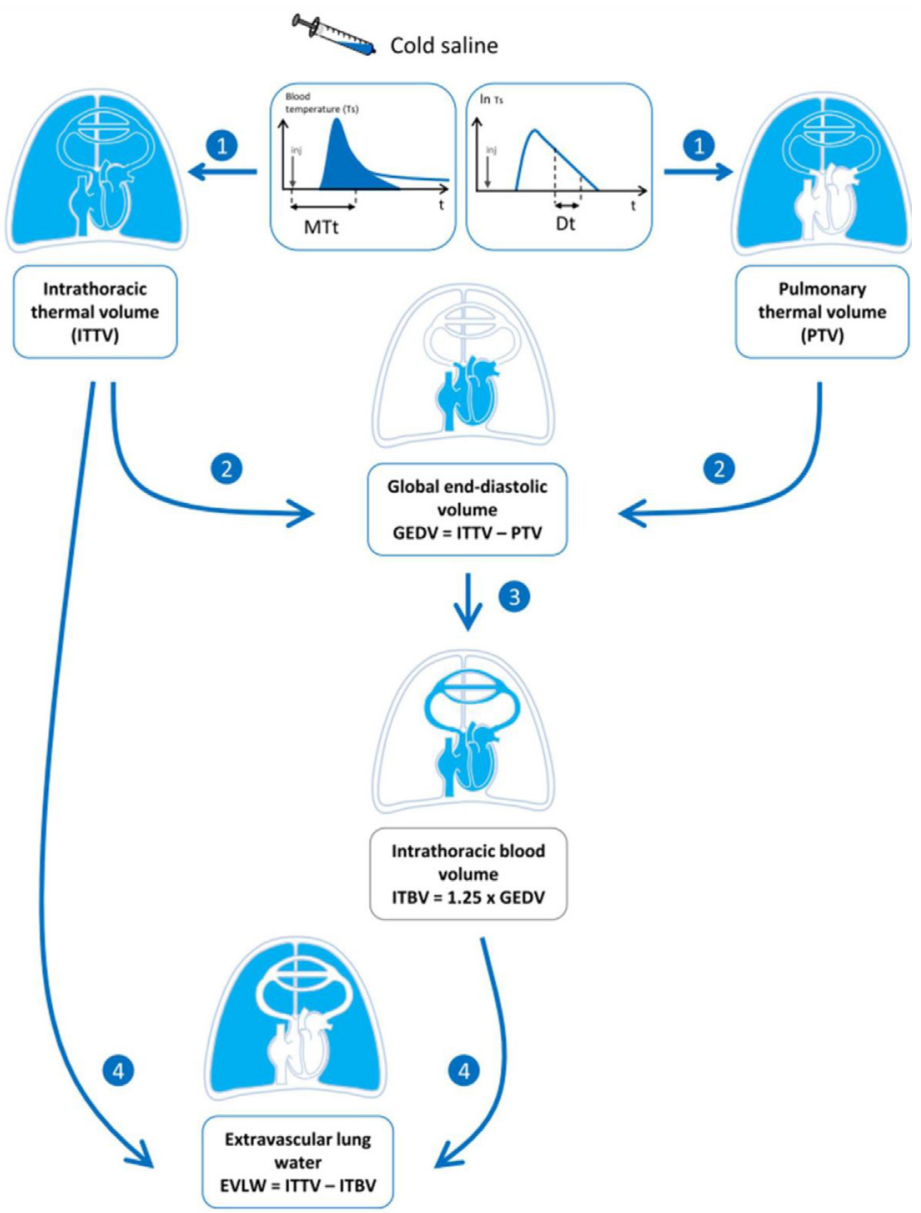

Fig. 2. Example of a volume estimation of intrathoracic compartments using TPTD. (Reproduced with permission from Monnet and Teboul Critical Care (2017) 21:147.)

associated with both hypovolemia and fluid overload, efforts have been made to find reliable predictors of fluid responsiveness, so that fluids can be administered only if an increase in $\mathrm{CO}$ is expected. In the past, filling pressures (CPV or PAOP) and volumetric variables (GEDV or intrathoracic blood volume) were used to estimate fluid responsiveness. However, studies have shown that those (static) indicators cannot reliably predict fluid responsiveness. ${ }^{68-72}$ Instead, there is increasing evidence that dynamic variables such as stroke volume variation and pulse pressure variation, which are based on the heart-lung interaction during mechanical ventilation, reliably predict fluid responsiveness. Dynamic variables are based on the changes in cardiac preload during different phases of the respiratory cycle, resulting in variations of stroke volume and pulse pressure. ${ }^{64,68,73-76}$ When intrathoracic pressure decreases during the ventilatory cycle, venous return increases, causing an increase in the stroke volume. In contrast, stroke volume decreases with the subsequent increase in intrathoracic pressure. Stroke volume variation is the maximum difference in stroke volume during a ventilatory cycle divided by the mean stroke volume. The pressure between the systolic and diastolic blood pressures is the pulse pressure. Pulse pressure variation is 
the maximum difference in pulse pressure during a ventilatory cycle divided by the mean pulse pressure. Patients who are fluid the responsive are on the steep part of the Frank-Starling curve and have high variations in stroke volume and pulse pressure during the ventilatory cycle (Fig. 3).

\section{Minimally Invasive Methods}

Stroke volume variation and pulse pressure variation are probably the most wellknown dynamic indices for predicting fluid responsiveness in mechanically ventilated patients. Both the stroke volume variation and the pulse pressure variation can be obtained with minimally invasive methods using an arterial catheter and monitor for pulse wave analysis ${ }^{64,68,73-76}$ or noninvasively using the volume clamp method. ${ }^{77}$ The use of stroke volume variation and pulse pressure variation is limited in several situations, including irregular heartbeats, spontaneously breathing patients or mechanical ventilation with low tidal volumes, open thorax, increased abdominal pressure, and a low heart rate to respiratory rate ratio. ${ }^{78}$ Previously, the variation of the systolic pressure during 1 mechanical respiratory cycle had been shown to predict hypovolemia and is calculated as the systolic arterial pressure during inspiration minus the systolic arterial pressure during expiration. ${ }^{79,80}$ The systolic pressure variation is not used in clinical routine anymore.

\section{Noninvasive Methods}

Noninvasive methods of assessing fluid responsiveness include noninvasively obtained stroke volume variation and pulse pressure variation using the volume clamp

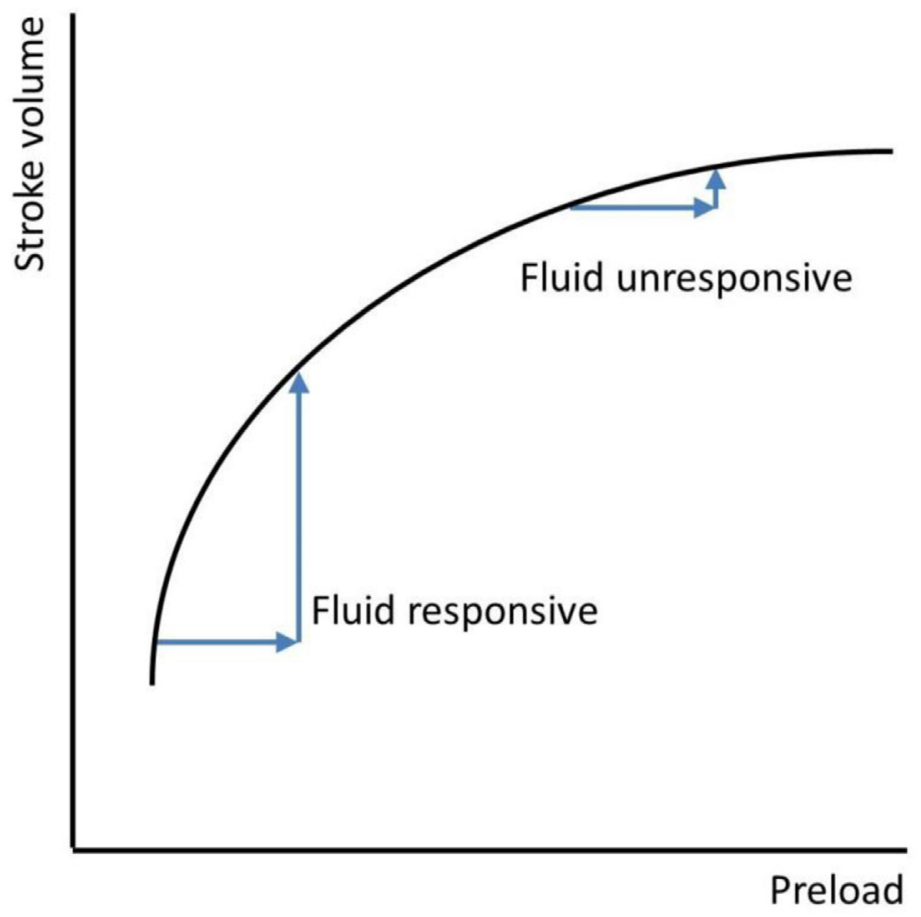

Fig. 3. Display of the fluid-responsive and fluid-unresponsive states of the left ventricle on the Frank-Starling curve. 


\begin{tabular}{|c|c|}
\hline \multicolumn{2}{|c|}{$\begin{array}{l}\text { Table } 3 \\
\text { Overview of dynamic indices calculations }\end{array}$} \\
\hline Dynamic Variable & Calculation \\
\hline Stroke volume variation & $S V V=\frac{S V V_{\max }-S V V_{\min }}{S V V_{\text {mean }}}$ \\
\hline Pulse pressure variation & $P P V=\frac{P P V_{\max }-P P V_{\min }}{P P V_{\text {mean }}}$ \\
\hline Systolic pressure variation & $S P V=S A P_{i n s p}-S A P_{\exp }$ \\
\hline Respiratory variations in $\triangle \mathrm{POP}$ & $\Delta P O P=\frac{P O P_{\max }-P O P_{\min }}{P O P_{\text {mean }}}$ \\
\hline PVI & $P V I=\frac{P I_{\max }-P I_{\min }}{P I_{\max }} \times 100$ \\
\hline
\end{tabular}

method, respiratory variations in pulse oximetry plethysmographic waveform amplitude $(\triangle \mathrm{POP})$, and automated pleth variability index $(\mathrm{PVI}){ }^{68,77,81}$ The volume clamp method was explained previously. Noninvasively obtained stroke volume variations and pulse pressure variations are acceptable predictors of fluid responsiveness. ${ }^{77}$ The $\triangle P O P$ is strongly correlated with the pulse pressure variation in mechanically ventilated patients ${ }^{82-84}$ and is able to predict fluid responsiveness in the operating room. ${ }^{81,84}$ The $\mathrm{PVI}$ is based on a commercial algorithm, which continuously assesses the $\triangle \mathrm{POP}$ using the perfusion index ${ }^{85}$ and has been shown to predict fluid responsiveness accurately. ${ }^{86,87}$ The use of $\triangle P O P$ and PVI might be limited during severe vasoconstriction $^{88}$ and in patients with a low perfusion index. ${ }^{89}$ An overview of the calculations used for the dynamic indices are given in Table 3. Other methods of assessing fluid responsiveness include echocardiographic measurements, such as the peak aortic flow velocity variation or vena cava collapsibility/distensibility, the passive leg raising test, the end-expiratory occlusion test, and others. ${ }^{68,90}$ Because these methods can only be performed intermittently, they are not further discussed in this review.

\section{DISCUSSION}

Many different tools are available for perioperative hemodynamic monitoring, both noninvasive and invasive, and a multitude of hemodynamic variables can be monitored. A profound knowledge of the methods and their limitations is required to choose the most appropriate monitoring device for the individual patient and indication. The type of perioperative hemodynamic monitoring should be chosen based on a thorough risk and benefit assessment, including the risk of the procedure and the risk of the individual patient, and this should be weighed against the risk of complications from the invasiveness of the monitoring method. The current review might guide the user in choosing the appropriate method.

\section{SUMMARY}

In this review, we aimed to provide an overview of hemodynamic monitoring methods used in the perioperative setting. Hemodynamic monitoring tools can be divided into 3 categories: invasive, minimally invasive, and noninvasive monitoring tools. The most invasive tool, the PAC, can be used to assess a multitude of hemodynamic variables, for example, $\mathrm{CO}$, pulmonary artery pressures, systemic vascular resistance, pulmonary vascular resistance, right ventricle end-diastolic volume or ejection fraction, 
and so on. However, owing to the risks associated with its invasiveness, the use of PAC is limited to select patient populations and procedures (mainly cardiac surgery). It remains unclear if PAC based treatment algorithms improve clinical outcomes. Slightly less invasive is the TPTD technique, which can be used to obtain the CO, GEDV, and EVLW. For arterial blood pressure monitoring, an arterial catheter remains the gold standard and pulse wave analysis can be used to estimate $\mathrm{CO}$, stroke volume, and dynamic indices (stroke volume variation and pulse pressure variation) for predicting fluid responsiveness. Pulse waves can also be derived and analyzed from the noninvasive continuous volume clamp method, which can provide the same hemodynamic variables. The CVP has been used for a long time for assessing fluid responsiveness; however, it has been shown to correlate poorly with the volume status of the patients, and therefore it has been proposed to use dynamic indices instead, obtained either invasively or noninvasively. Besides stroke volume variation and pulse pressure variation, the $\triangle \mathrm{POP}$ and $\mathrm{PVI}$ can be used to predict fluid responsiveness in the operating room. The $P_{\mathrm{msf}}$ can be used to accurately assess a patient's volume status, although it is quite difficult to measure and, thus, is of limited clinical use.

\section{CLINICS CARE POINTS}

- The CVP should not be used to assess preload or fluid responsiveness; dynamic variables should be used instead.

- The $P_{\mathrm{ms}}$, although difficult to measure, can be used to accurately assess a patient's volume status.

- CO can be monitored using different methods with different levels of invasiveness and risks of complications. The best suited type of monitoring should be determined for every individual patient and procedure.

\section{DISCLOSURE}

I.N. de Keijzer has nothing to disclose. T.W.L. Scheeren received research grants and honoraria from Edwards Lifesciences (Irvine, California, USA) and Masimo (Irvine, CA) for consulting and lecturing and from Pulsion Medical Systems SE (Feldkirchen, Germany) for lecturing.

\section{REFERENCES}

1. Dalley S. Myths from Mesopotamia: creation, the flood, Gilgamesh and others. Oxford (England, UK): Oxford University Press; 1989.

2. Ghasemzadeh N, Zafari AM. A brief journey into the history of the arterial pulse. Cardiol Res Pract 2011;2011:164832.

3. Bolli R. William Harvey and the Discovery of the Circulation of the Blood. Circ Res 2019;124:1169-71.

4. Esunge PM. From blood pressure to hypertension: the history of research. J R Soc Med 1991;84:621.

5. Neil E. Carl Ludwig and His Pupils. Circ Res 1961;IX:971-8.

6. Roguin A. Scipione Riva-Rocci and the men behind the mercury sphygmomanometer. Int J Clin Pract 2006;60:73-9.

7. Wesseling K. A century of non-invasive arterial pressure measurement: form Marey to Peñáz and Finapres. Homeost Heal Dis 1995;36:50-66. 
8. Einthoven W. Un nouveau galvanomètre. Arch Neerl Sc Ex Nat 1901;6:625-33.

9. Burnett J. The origins of the electrocardiograph as a clinical instrument. Med Hist 1985;(Supplement 5):53-76.

10. Saugel B, Sessler DI. Perioperative Blood Pressure Management. Anesthesiology 2021;134:250-61.

11. Saugel B, Kouz K, Meidert AS, et al. How to measure blood pressure using an arterial catheter: a systematic 5-step approach. Crit Care 2020;24:172.

12. Handlogten KS, Wilson GA, Clifford L, et al. Brachial artery catheterization: an assessment of use patterns and associated complications. Anesth Analg 2014; 118:288-95.

13. Scheer BV, Perel A, Pfeiffer UJ. Clinical review: complications and risk factors of peripheral arterial catheters used for haemodynamic monitoring in anaesthesia and intensive care medicine. Crit Care 2002;6:198-204.

14. Roach JK, Thiele RH. Perioperative blood pressure monitoring. Best Pract Res Clin Anaesthesiol 2019;33:127-38.

15. Forouzanfar M, Dajani H, Groza V, et al. Oscillometric blood pressure estimation: past, present, and future. IEEE Rev Biomed Eng 2015;8:44-63.

16. Yelderman M, Ream A. Indirect measurement of mean blood pressure in the anesthetized patient. Anesthesiology 1979;50:253-6.

17. Kouz K, Scheeren TWL, de Backer D, et al. Pulse Wave Analysis to Estimate Cardiac Output. Anesthesiology 2020;134:119-26.

18. Saugel B, Hoppe P, Nicklas JY, et al. Continuous noninvasive pulse wave analysis using finger cuff technologies for arterial blood pressure and cardiac output monitoring in perioperative and intensive care medicine: a systematic review and meta-analysis. Br J Anaesth 2020;125:25-37.

19. Saugel B, Kouz K, Scheeren TWL, et al. Cardiac output estimation using pulse wave analysis-physiology, algorithms, and technologies: a narrative review. $\mathrm{Br}$ J Anaesth 2021;126:67-76.

20. Rogge DE, Nicklas JY, Schön G, et al. Continuous noninvasive arterial pressure monitoring in obese patients during bariatric surgery: an evaluation of the vascular unloading technique. Anesth Analg 2019;128:477-83.

21. Berkelmans GFN, Kuipers S, Westerhof BE, et al. Comparing volume-clamp method and intra-arterial blood pressure measurements in patients with atrial fibrillation admitted to the intensive or medium care unit. J Clin Monit Comput 2018;32:439-46.

22. Shah $P$, Louis M. Physiology, central venous pressure.. In: StatPearls [Internet]. 2020. Available at: https://www.ncbi.nlm.nih.gov/books/NBK519493/. Accessed November 19, 2020.

23. Magder S. How to use central venous pressure measurements. Curr Opin Crit Care 2005;11:264-70.

24. Guyton A. Determination of cardiac output by equating venous return curves with cardiac response curves. Physiol Rev 1955;35:123-9.

25. Kumar A, Anel R, Bunnell E, et al. Pulmonary artery occlusion pressure and central venous pressure fail to predict ventricular filling volume, cardiac performance, or the response to volume infusion in normal subjects. Crit Care Med 2004;32:691-9.

26. Boldt J, Lenz M, Kumle B, et al. Volume replacement strategies on intensive care units: results from a postal survey. Intensive Care Med 1998;24:147-51.

27. Michard F, Alaya S, Zarka V, et al. Global end-diastolic volume as an indicator of cardiac preload in patients with septic shock. Chest 2003;124:1900-8. 
28. Hofer CK, Furrer L, Matter-Ensner S, et al. Volumetric preload measurement by thermodilution: a comparison with transoesophageal echocardiography. $\mathrm{Br} J$ Anaesth 2005;94:748-55.

29. Marik PE, Baram M, Vahid B. Does central venous pressure predict fluid responsiveness? A Systematic Review of the Literature and the Tale of Seven Mares. Chest 2008;134:172-8.

30. Marik PE, Cavallazzi R. Does the central venous pressure predict fluid responsiveness? An updated meta-analysis and a plea for some common sense. Crit Care Med 2013;41:1774-81.

31. Pinsky M, Kellum J, Bellomo R. Central venous pressure is a stopping rule, not a target of fluid resuscitation. Crit Care Resusc 2015;17:56.

32. Van Beest P, Wietasch $G$, Scheeren T, et al. Clinical review: use of venous oxygen saturations as a goal - a yet unfinished puzzle. Crit Care 2011;15:232.

33. Vincent JL. The pulmonary artery catheter. J Clin Monit Comput 2012;26:341-5.

34. Bootsma I, Boerma E, de Lange F, et al. The contemporary pulmonary artery catheter. Part 1: placement and waveform analysis. J Clin Monit Comput 2021. https://doi.org/10.1007/s10877-021-00662-8.

35. O'Quin R, Marini J. Pulmonary artery occlusion pressure: clinical physiology, measurement, and interpretation. Br J Psychother 1983;128:319-26.

36. Shah M, Hasselblad V, Stevenson L, et al. Impact of the pulmonary artery catheter in critically ill patients: meta-analysis of randomized clinical trials. JAMA 2005; 294:1664-70.

37. Rajaram S, Desai N, Kalra A, et al. Pulmonary artery catheters for adult patients in intensive care (Review). Cochrane Database Syst Rev 2013;2:CD003408.

38. Harvey S, Harrison DA, Singer M, et al. Assessment of the clinical effectiveness of pulmonary artery catheters in management of patients in intensive care (PACMan): a randomised controlled trial. Lancet 2005;366:472-7.

39. Cohen MG, Kelly RV, Kong DF, et al. Pulmonary artery catheterization in acute coronary syndromes: insights from the GUSTO IIb and GUSTO III trials. Am J Med 2005;118:482-8.

40. Rapoport J, Teres D, Steingrub J, et al. Patient characteristics and ICU organizational factors that influence frequency of pulmonary artery catheterization. J Am Med Assoc 2000;283:2559-67.

41. Koo KKY, Sun JCJ, Zhou Q, et al. Pulmonary artery catheters: evolving rates and reasons for use. Crit Care Med 2011;39:1613-8.

42. Ospina-Tascón GA, Cordioli RL, Vincent JL. What type of monitoring has been shown to improve outcomes in acutely ill patients? Intensive Care Med 2008; 34:800-20.

43. Rothe CF. Mean circulatory filling pressure: its meaning and measurement. J Appl Physiol 1993;74:499-509.

44. Maas JJ, Pinsky MR, Geerts BF, et al. Estimation of mean systemic filling pressure in postoperative cardiac surgery patients with three methods. Intensive Care Med 2012;38:1452-60.

45. Wijnberge M, Sindhunata DP, Pinsky MR, et al. Estimating mean circulatory filling pressure in clinical practice: a systematic review comparing three bedside methods in the critically ill. Ann Intensive Care 2018;8:73.

46. Maas JJ, Geerts BF, Van Den Berg PCM, et al. Assessment of venous return curve and mean systemic filling pressure in postoperative cardiac surgery patients. Crit Care Med 2009;37:912-8.

47. Fessler HE, Brower RG, Wise RA, et al. Effects of positive end-expiratory pressure on the canine venous return curve. Am Rev Respir Dis 1991;143:19-24. 
48. Parkin WG, Leaning MS. Therapeutic control of the circulation. J Clin Monit Comput 2008;22:391-400.

49. Guyton A, Lindsey A, Abernathy B, et al. Venous return at various right atrial pressures and the normal venous return curve. Am J Physiol 1957;189:609-15.

50. Saugel B, Vincent JL. Cardiac output monitoring: how to choose the optimal method for the individual patient. Curr Opin Crit Care 2018;24:165-72.

51. Monnet X, Teboul JL. Transpulmonary thermodilution: advantages and limits. Crit Care 2017;21:147.

52. Reuter DA, Huang C, Edrich T, et al. Cardiac output monitoring using indicatordilution techniques: basics, limits, and perspectives. Anesth Analg 2010;110: 799-811.

53. Jozwiak M, Monnet X, Teboul J-L. Pressure waveform analysis. Anesth Analg 2018;126:1930-3.

54. Esper SA, Pinsky MR. Arterial waveform analysis. Best Pract Res Clin Anaesthesiol 2014;28:363-80.

55. Slagt C, Malagon I, Groeneveld ABJ. Systematic review of uncalibrated arterial pressure waveform analysis to determine cardiac output and stroke volume variation. Br J Anaesth 2014;112:626-37.

56. Saugel B, Cecconi M, Wagner JY, et al. Noninvasive continuous cardiac output monitoring in perioperative and intensive care medicine. $\mathrm{Br} J$ Anaesth 2015; 114:562-75.

57. Appel P, Kram H, Mackabee J, et al. Comparison of measurements of cardiac output by bioimpedance and thermodilution in severely ill surgical patients. Crit Care Med 1986;14:933-5.

58. Marik P, Pendelton J, Smith R. A comparison of hemodynamic parameters derived from transthoracic electrical bioimpedance with those parameters obtained by thermodilution and ventricular angiography. Crit Care Med 1997;25: $1545-50$.

59. Sageman W, Amundson D. Thoracic electrical bioimpedance measurement of cardiac output in postaortocoronary bypass patients. Crit Care Med 1993;21: 1139-42.

60. Chamos C, Vele L, Hamilton M, et al. Less invasive methods of advanced hemodynamic monitoring: principles, devices, and their role in the perioperative hemodynamic optimization. Perioper Med 2013;2:19.

61. Critchley LAH, Calcroft RM, Tan PYH, et al. The effect of lung injury and excessive lung fluid, on impedance cardiac output measurements, in the critically ill. Intensive Care Med 2000;26:679-85.

62. Wallace AW, Salahieh A, Lawrence A, et al. Endotracheal cardiac output monitor. Anesthesiology 2000;92:178-89.

63. Marik PE. Noninvasive cardiac output monitors: a state-of the-art review. J Cardiothorac Vasc Anesth 2013;27:121-34.

64. Marik PE, Cavallazzi R, Vasu T, et al. Dynamic changes in arterial waveform derived variables and fluid responsiveness in mechanically ventilated patients: a systematic review of the literature. Crit Care Med 2009;37:2642-7.

65. Jozwiak M, Teboul JL, Monnet X. Extravascular lung water in critical care: recent advances and clinical applications. Ann Intensive Care 2015;5:38.

66. Sakka SG, Rühl CC, Pfeiffer UJ, et al. Assessment of cardiac preload and extravascular lung water by single transpulmonary thermodilution. Intensive Care Med 2000;26:180-7.

67. Marik PE. Fluid Responsiveness and the Six Guiding Principles of Fluid Resuscitation. Crit Care Med 2016;44:1920-2. 
68. Renner J, Scholz J, Bein B. Monitoring fluid therapy. Best Pract Res Clin Anaesthesiol 2009;23:159-71.

69. Michard F, Teboul JL. Predicting fluid responsiveness in ICU patients: a critical analysis of the evidence. Chest 2002;121:2000-8.

70. Bendjelid K, Romand JA. Fluid responsiveness in mechanically ventilated patients: a review of indices used in intensive care. Intensive Care Med 2003; 29(3):352-60.

71. Calvin J, Driedger A, Sibbald W. The hemodynamic effect of rapid fluid infusion in critically ill patients. Surgery 1981;90:61-76.

72. Michard F, Boussat S, Chemla D, et al. Relation between respiratory changes in arterial pulse pressure and fluid responsiveness in sepsis. Am J Respir Crit Care Med 2000;162:134-8.

73. Carsetti A, Cecconi M, Rhodes A. Fluid bolus therapy: monitoring and predicting fluid responsiveness. Curr Opin Crit Care 2015;21:388-94.

74. Guerin L, Monnet X, Teboul JL. Monitoring volume and fluid responsiveness: from static to dynamic indicators. Best Pract Res Clin Anaesthesiol 2013;27:177-85.

75. Jozwiak M, Monnet X, Teboul J-L. Prediction of fluid responsiveness in ventilated patients. Ann Transl Med 2018;6:352.

76. Monnet X, Marik PE, Teboul JL. Prediction of fluid responsiveness: an update. Ann Intensive Care 2016;6:111.

77. Vos JJ, Poterman M, Salm PP, et al. Noninvasive pulse pressure variation to predict fluid responsiveness at multiple thresholds: a prospective observational study. Can J Anaesth 2015;62:1153-60.

78. Michard F, Chemla D, Teboul JL. Applicability of pulse pressure variation: how many shades of grey? Crit Care 2015;19:144.

79. Kramer A, Zygun D, Hawes $H$, et al. Pulse pressure variation predicts fluid responsiveness following coronary artery bypass surgery. Chest 2004;126: 1563-8.

80. Tavernier B, Makhotine O, Lebuffe G, et al. Systolic pressure variation as a guide to fluid therapy in patients with sepsis-induced hypotension. Anesthesiology 1998;89:1313-21.

81. Cannesson M, Attof $Y$, Rosamel $P$, et al. Respiratory variations in pulse oximetry plethysmographic waveform amplitude to predict fluid responsiveness in the operating room. Anesthesiology 2007;106:1105-11.

82. Cannesson M, Besnard C, Durand PG, et al. Relation between respiratory variations in pulse oximetry plethysmographic waveform amplitude and arterial pulse pressure in ventilated patients. Crit Care 2005;9:R562-8.

83. Natalini G, Rosano A, Franceschetti M, et al. Variations in arterial blood pressure and photoplethysmography during mechanical ventilation. Anesth Analg 2006; 103:1182-8.

84. Solus-Biguenet H, Fleyfel M, Tavernier B, et al. Non-invasive prediction of fluid responsiveness during major hepatic surgery. Br J Anaesth 2006;97:808-16.

85. Cannesson M, Delannoy B, Morand A, et al. Does the pleth variability index indicate the respiratory- induced variation in the plethysmogram and arterial pressure waveforms? Anesth Analg 2008;106:1189-94.

86. Cannesson M, Desebbe $\mathrm{O}$, Rosamel $\mathrm{P}$, et al. Pleth variability index to monitor the respiratory variations in the pulse oximeter plethysmographic waveform amplitude and predict fluid responsiveness in the operating theatre. $\mathrm{Br} J$ Anaesth 2008;101:200-6.

87. Zimmermann M, Feibicke T, Keyl C, et al. Accuracy of stroke volume variation compared with pleth variability index to predict fluid responsiveness in 
mechanically ventilated patients undergoing major surgery. Eur J Anaesthesiol 2010;27:555-61.

88. Perel A. Automated assessment of fluid responsiveness in mechanically ventilated patients. Anesth Analg 2008;106:1031-3.

89. Broch $\mathrm{O}$, Bein B, Gruenewald M, et al. Accuracy of the pleth variability index to predict fluid responsiveness depends on the perfusion index. Acta Anaesthesiol Scand 2011;55:686-93.

90. Aditianingsih D, George YWH. Guiding principles of fluid and volume therapy. Best Pract Res Clin Anaesthesiol 2014;28:249-60. 